\title{
Study Of Superconductivity And Antiferromagnetism In Rare Earth Nickel Borocarbides $\left(\mathrm{RNi}_{2} \mathrm{~B}_{2} \mathrm{C}\right)$
}

\author{
${ }^{1}$ Dr. Salila Das and Preeti Suman Dash \\ ${ }^{I}$ Department of Physics, Berhampur University,India.
}

\begin{abstract}
We have reported theoretically the coexistence of antiferromagnetism and superconductivity in rare earth nickel borocarbide compounds. By using a suitable model Hamiltonian we have derived critical temperature, zero order parameter, gap to $T_{c}$ ratio. We have shown that the critical temperature is decreased as hybridization coefficient increases. We have also calculated the effect of external magnetic field on the coexisting state of superconductivity and antiferromagnetism. Our numerical calculations show an interplay between the two order parameters and are able to explain some of the experimental results reported for $\left(\mathrm{RNi}_{2} \mathrm{~B}_{2} \mathrm{C}\right)$ superconductors.
\end{abstract}

Keywords:Superconductivity; Antiferromagnetism; borocarbide

\section{Introduction}

The coexistence of antiferromagnetism (AFM) and superconducting(SC) properties of rare earth nickel borocarbide compounds $\left(R N i_{2} B_{2} C\right.$ ) where $\mathrm{R}=\mathrm{Y}, \mathrm{Lu} \mathrm{Dy}, \mathrm{Ho}, \mathrm{Er}$, and $\mathrm{Tm}$ motivate the researchers to go deep into their electronic structure as well as detail investigation of the interplay between SC and AFM states.The coexistence properties of these compounds have become a great interest for the theoretical and experimental researchers [1-13]. It has been seen that these compounds have layered structure made up of rare-earth carbon (R-C) sheets with the stacking of $\mathrm{Ni}_{2} \mathrm{~B}$ slabs along c-axis. The band structure of borocarbide $\mathrm{RNi}_{2} \mathrm{~B}_{2} \mathrm{C}$ exhibit four bands crossing the Fermi level. One of the four bands is flat which indicates the strong correlation of the system. The flat band hybridizes with other conducting bands. Due to this hybridization, the AFM of the sublattice acquires the localized state of the d-band.There exists a peak density of states at the Fermi-level where $\mathrm{d}$ band of $\mathrm{Ni}$ and other bands, carbon, boron and rare earth compound contribute[4,5]. The localized $4 \mathrm{f}$ electrons of the rare earth are mostly responsible for the anisotropic magnetic properties. On the other hand, the itinerant electrons responsible for superconductivity which arises from spin wave due to Fermi surface instability also contribute to antiferromagnetism. These borocarbide compounds exhibit well-established coexistence of superconductivity and magnetism [1,2] with a number of interesting properties. However, a detailed understanding of how magnetism affects superconductivity still needs to be accomplished.

The rare earth borocarbide series is unique as its members have all the conceivable combinations of $T_{c} \geq T_{N}$ for $\operatorname{ErNi}_{2} B_{2} C\left(T_{N}=6 k\right.$ and $\left.T_{c}=11 k\right)$ and $T m N i_{2} B_{2} C\left(T_{N}=1.5 k\right.$ and $\left.T_{c}=10.5 \mathrm{~K}\right), T_{c}$ $\equiv T_{N}$ for $\mathrm{HoNi}_{2} \mathrm{~B}_{2} \mathrm{C}\left(T_{N}, T_{c}=8.5 \mathrm{k}\right)$ and $T_{c} \leq T_{N}$ for $D y N i_{2} B_{2} C \quad\left(T_{N}=10.5 \mathrm{k}\right.$ and $\left.T_{c}=6.5 \mathrm{k}\right)$ [7,20]. Point contact measurement of the superconducting energy gap in Y[21] and Ho[22] provides $2 \Delta_{0} / k_{B} T_{c}$ $=3.63 \pm 0.05$. The point contact techniques based on Andreev reflection from a normal superconducting boundary, reported the values of $2 \Delta_{0} / k_{B} T_{c}$ for $D y N i_{2} B_{2} C$ as 3.6[22]. Similarly, $2 \Delta_{0} / k_{B} T_{c}$ value for $\mathrm{ErNi}_{2} \mathrm{~B}_{2} \mathrm{C}$ obtained from point contact spectra of the Andreev reflection curves is 3.6 [23]. In this paper, our aim is to calculate the gap parameter of nickel borocarbide compound in presence of magnetic field by applying the two-band model. Both Amici et.al.[19] and Sahu et. al[24] investigated some of the properties of these compounds by taking CDW on BCS superconductors within a mean field approximation. In this paper, we have modified the Hamiltonian of Sahu et. al. by taking hybridization in presence of external magnetic field. Some physical properties like $T_{C}, T_{N}$, order parameter, gap to $T_{C}$ ratio were investigated which agrees quite well with the experimental results. 


\section{Theoretical Model}

To study the interplay of superconductivity and antiferromagnetism we consider a model Hamiltonian [16] in which besides pairing interaction among conduction electrons, there exists effective antiferromagnetic coupling between nearest neighbour localized electron and the interaction between conduction and localized electrons. The antiferromagnetic exchange leads to Neel ground state which is characterized by antiferromagnetic order. The different interactions taken in our model Hamiltonian is given by

$$
H=H_{0}+H_{s}+H_{v}+H_{h}+H_{f}+H_{B}
$$

The Hamiltonian $H_{o}$ involving hopping of conduction electrons between two neighbouring sites is taken as

$$
H_{o}=\sum_{\mathbf{k} \sigma} \varepsilon_{o}(\mathbf{k})\left(a_{\mathbf{k} \sigma}^{\dagger} b_{\mathbf{k} \sigma}+\text { h.c. }\right)
$$

where $a_{\mathbf{k} \sigma}^{\dagger}, b_{\mathbf{k} \sigma}^{\dagger}$ are the creation operators of electrons at sites 1 and 2 for conduction electrons and $a_{k \sigma}, b_{k \sigma}$ are the corresponding annihilation operators. The dispersion energy $\varepsilon_{o}(\mathbf{k})$ is given by

$\varepsilon_{o}(\mathbf{k})=-2 t\left(\cos k_{x}+\cos k_{y}\right)$

The mean field BCS Hamiltonian describing phonon mediated superconductivity is given by

$$
H_{s}=-\Delta \sum_{\mathbf{k}}\left[\left(a_{\mathbf{k} \uparrow}^{\dagger} a_{-\mathbf{k} \downarrow}^{\dagger}+\text { h.c. }\right)+\left(b_{\mathbf{k} \uparrow}^{\dagger} b_{-\mathbf{k} \downarrow}^{\dagger}+\text { h.c. }\right)\right](3)
$$

Here only intra sublattice Cooper pairing is assumed and the superconducting gap parameter $(\Delta)$ is defined as

$$
\Delta=-\sum_{k} \tilde{V}_{k}\left(<a_{k \uparrow}^{\dagger} a_{-k \downarrow}^{\dagger}>+<b_{k \uparrow}^{\dagger} b_{-k \downarrow}^{\dagger}>\right)(4)
$$

$\tilde{V}_{k}$ is the strength of attractive interaction between two electrons via phonon. Effective hybridization between the f-electrons and the conduction electrons contributes to the Hamiltonian $H_{v}$ i.e, given by

$$
H_{v}=V \sum_{\mathbf{k} \sigma}\left(a_{\mathbf{k} \sigma}^{\dagger} f_{1, \mathbf{k} \sigma}+b_{\mathbf{k} \sigma}^{\dagger} f_{2, \mathbf{k} \sigma}+\text { h.c. }\right)
$$

where $V$ is the hybridization interaction constant $f_{i, \mathbf{k} \sigma}^{\dagger}$ and $f_{i, \mathbf{k} \sigma}(\mathrm{i}=1,2)$ are the creation and annihilation operators, respectively at sites $\mathrm{i}$ of $\mathrm{f}$ electrons. Strongly antiferromagnetic correlation and conduction of $\mathrm{Ni}$ d-electrons is stimulated by a staggered magnetic field of strength $\mathrm{h}$ and this contributes to the Hamiltonian $H_{h}$ is written as

$$
H_{h}=\left(\frac{h}{2}\right) \sum_{\mathbf{k} \sigma} \sigma\left(a_{\mathbf{k} \sigma}^{\dagger} a_{\mathbf{k} \sigma}-b_{\mathbf{k} \sigma}^{\dagger} b_{\mathbf{k} \sigma}\right)
$$

The intra f-electron Hamiltonian (for the electron in the localized levels corresponding to the flat band) in the presence of external magnetic field B is

$$
H_{f}=\left(\varepsilon_{f}+\frac{1}{2} g \mu_{B} B\right) \sum_{\mathbf{k} \sigma} f_{i, \mathbf{k} \uparrow}^{\dagger} f_{i, \mathbf{k} \uparrow}+\left(\varepsilon_{f}-\frac{1}{2} g \mu_{B} B\right) \sum_{\mathbf{k} \sigma} f_{i, \mathbf{k} \downarrow}^{\dagger} f_{i, \mathbf{k} \downarrow}
$$

where $\varepsilon_{f}$ is the renormalized f-level energy. g and $\mu_{B} B$ are Lande g factor and Bohr magneton respectively. 
The external magnetic field contributes to the Hamiltonian $H_{B}$ is written as

$$
H_{B}=\sigma g \mu_{B} B \sum_{\mathbf{k} \sigma}\left(a_{\mathbf{k} \sigma}^{\dagger} a_{\mathbf{k} \sigma}+b_{\mathbf{k} \sigma}^{\dagger} b_{\mathbf{k} \sigma}\right)
$$

Here $\mathrm{B}$ is the external magnetic field.

Each band is a function of staggered magnetic field(h), the superconducting gap parameter $\Delta$ ) and strength of hybridization(v). Here we have solved the Green's function to calculate the appropriate single particle correlation function which in turn determines the order parameter corresponding to superconducting and antiferromagnetic long-range order. These are given by

$$
\Delta_{k}(T)=-\Sigma_{k} \tilde{V}_{k}\left[\left\langle a_{\mathbf{k} \uparrow}^{\dagger} a_{-\mathbf{k} \downarrow}^{\dagger}>+<b_{\mathbf{k} \uparrow}^{\dagger} b_{-\mathbf{k} \downarrow}^{\dagger}>\right](9)\right.
$$

and

$$
h=-\frac{1}{2} g \mu_{B} \sum_{\mathbf{k}}\left[\left\{\left\langlea_{\mathbf{k} \uparrow}^{\dagger} a_{\mathbf{k} \uparrow}>-\left\langle b_{\mathbf{k} \uparrow}^{\dagger} b_{\mathbf{k} \uparrow}>\right\}-\left\{\left\langle a_{\mathbf{k} \downarrow}^{\dagger} a_{\mathbf{k} \downarrow}>-<b_{\mathbf{k} \downarrow}^{\dagger} b_{\mathbf{k} \downarrow}>\right\}\right]\right.\right.\right.
$$

After calculation we have

$$
\Delta_{\mathbf{k}}(T)=-V_{0} N(0) \int_{-\omega_{D}}^{+\omega_{D}} d \varepsilon_{0}(\mathbf{k})\left[F_{1}(\mathbf{k}, T)+F_{2}(\mathbf{k}, T)\right]
$$

and

$$
h=\frac{N(0)}{2} g \mu_{B} \int_{-\frac{\omega}{2}}^{+\frac{\omega}{2}}\left[\left(F_{1}(k, T)-F_{2}(k, T)\right)+\left(F_{3}(k, T)-F_{4}(k, T)\right)\right]
$$

where

$$
\begin{aligned}
& F_{1}(\mathbf{k}, T)=\frac{\Delta_{-} e^{\frac{-\beta \alpha}{2}}}{\omega_{1}^{\prime 2}-\omega_{2}^{\prime 2}}\left\{\left\{\omega_{1}^{\prime}\left(\frac{1}{e^{\beta \omega_{1}^{\prime}}+e^{-\frac{\beta \alpha}{2}}}-\frac{1}{e^{-\beta \omega_{1}^{\prime}}+e^{-\frac{\beta \alpha}{2}}}\right)\right\}\right. \\
& \left.-\left\{\omega_{2}^{\prime}\left(\frac{1}{e^{\beta \omega_{2}^{\prime}}+e^{-\frac{\beta \alpha}{2}}}-\frac{1}{e^{-\beta \omega_{2}^{\prime}}}+e^{-\frac{\beta \alpha}{2}}\right)\right\}\right] \\
& F_{2}(\mathbf{k}, T)=\frac{\Delta_{+} e^{\frac{-\beta \alpha}{2}}}{\omega_{3}^{\prime 2}-\omega_{4}^{\prime 2}}\left[\left\{\omega_{3}^{\prime}\left(\frac{1}{e^{\beta \omega_{3}^{\prime}}+e^{-\frac{\beta \alpha}{2}}}-\frac{1}{e^{-\beta \omega_{3}^{\prime}}+e^{-\frac{\beta \alpha}{2}}}\right)\right\}\right. \\
& \left.-\left\{\omega_{4}^{\prime}\left(\frac{1}{e^{\beta \omega_{4}^{\prime}}+e^{-\frac{\beta \alpha}{2}}}-\frac{1}{e^{-\beta \omega_{4}^{\prime}}+e^{-\frac{\beta \alpha}{2}}}\right)\right\}\right], \\
& F_{3}(\mathbf{k}, T)=\frac{\Delta_{-} e^{\frac{-\beta \alpha}{2}}}{\omega_{1}^{\prime 2}-\omega_{2}^{\prime 2}}\left\{\left\{\omega_{1}^{\prime \prime}\left(\frac{1}{e^{\beta \omega_{1}^{\prime \prime}}+e^{-\frac{\beta \alpha}{2}}}-\frac{1}{e^{-\beta \omega_{1}^{\prime \prime}}+e^{-\frac{\beta \alpha}{2}}}\right)\right\}\right. \\
& \left.-\left\{\omega_{2}^{\prime \prime}\left(\frac{1}{e^{\beta \omega_{2}^{\prime \prime}}+e^{-\frac{\beta \alpha}{2}}}-\frac{1}{e^{-\beta \omega_{2}^{\prime \prime}}+e^{-\frac{\beta \alpha}{2}}}\right)\right\}\right] \text {, }
\end{aligned}
$$

and 


$$
\begin{aligned}
& F_{4}(\mathbf{k}, T)=\frac{\Delta_{+} e^{\frac{-\beta \alpha}{2}}}{\omega_{3}^{\prime 2}-\omega_{4}^{\prime 2}}\left[\left\{\omega_{3}^{\prime \prime}\left(\frac{1}{e^{\beta \omega_{3}^{\prime \prime}}+e^{-\frac{\beta \alpha}{2}}}-\frac{1}{e^{-\beta \omega_{3}^{\prime \prime}}+e^{-\frac{\beta \alpha}{2}}}\right)\right\}\right. \\
& \left.-\left\{\omega_{4}^{\prime \prime}\left(\frac{1}{e^{\beta \omega_{4}^{\prime \prime}}+e^{-\frac{\beta \alpha}{2}}}-\frac{1}{e^{-\beta \omega_{4}^{\prime \prime}}+e^{-\frac{\beta \alpha}{2}}}\right)\right\}\right]
\end{aligned}
$$

where $\mathrm{N}(0)$ is the density of states of the conduction electrons at the Fermi level $\varepsilon_{F}$. All the parameters used in the above equations are divided by $2 \mathrm{t}$ to make them dimensionless, where $\mathrm{W}=8 \mathrm{t}$, is the width of the conduction band. We have

$$
\begin{aligned}
& \frac{\Delta(T)}{2(t)}=z, \frac{\omega_{D}}{2(t)}=\tilde{\omega}_{D}, \frac{k_{\beta}}{2(t)}=\theta \\
& \frac{\boldsymbol{W}}{2 t}=\frac{\tilde{W}}{2}, \frac{\boldsymbol{h}}{2(\boldsymbol{t})}=\boldsymbol{h} \\
& \frac{V}{2(t)}=V, \frac{\varepsilon_{0}(\mathbf{k})}{2(t)}=x_{0} \\
& \frac{N(0) V_{0}}{2(t)}=\lambda_{1}, \frac{\frac{1}{2} N(0) g \mu_{B}}{2(t)}=\lambda_{2}(16)
\end{aligned}
$$

The superconducting and staggered magnetic field gap equation in dimensionless form are

$z=\lambda_{1} \int_{-\tilde{w}_{D}}^{+\tilde{w}_{D}} d x_{0}(\mathbf{k})\left[F_{1}\left(x_{0}, \theta\right)+F_{2}\left(x_{0}, \theta\right)\right]$

and

$h=\lambda_{2} \int_{\frac{-\widetilde{W}}{2}}^{\frac{\widetilde{W}}{2}}\left[\left(F_{1}\left(x_{0}, \theta\right)-F_{2}\left(x_{0}, \theta\right)\right)+\left(F_{3}\left(x_{0}, \theta\right)-F_{4}\left(x_{0}, \theta\right)\right)\right]$

We have considered the half-filled band situation (the Fermi level lie in the middle of the antiferromagnetic band gap) where the Fermi level is taken as zero $\left(\varepsilon_{F}=0\right)$. Our eqn. (33) and (34) are coupled equations and have to be solved self-consistently in order to study the temperature variation of any of these two quantities which are necessary to understand the nature of the co-existence phase.

\section{Discussions}

Here we have solved antiferromagnetic order parameter along with the gap parameter self-consistently and numerically for the compounds $\mathrm{HoNi}_{2} \mathrm{~B}_{2} \mathrm{C}, \mathrm{DyNi} \mathrm{B}_{2} \mathrm{~B}, \mathrm{ErNi}_{2} \mathrm{~B}_{2} \mathrm{C}, \mathrm{TmNi} \mathrm{i}_{2} \mathrm{~B}_{2} \mathrm{C}$. A standard set of dimensionless parameters $[16,24]$ are used in the calculations with bandwidth, $W=1 \mathrm{eV}$ and $\omega_{D}=8 \mathrm{t}$, where $\mathrm{t}$ is the hopping integral.

Fig-1 shows mean field behaviour of superconducting and antiferromagnetic order parameter for $\mathrm{HoNi}_{2} \mathrm{~B}_{2} \mathrm{C}$ $\left.T_{c}=9.5\right), D y N i_{2} B_{2} C\left(T_{c}=8.98\right), E r N i_{2} B_{2} C\left(T_{c}=7.8\right), T m N i_{2} B_{2} C\left(T_{c}=7.8\right)$ in the absence of an external magnetic field.

Fig-2 shows superconducting order parameter along with antiferromagnetic order parameter for $\mathrm{HoNi}_{2} \mathrm{~B}_{2} \mathrm{C}$ the compound in presence of external magnetic field. The critical temperature parameter $\theta_{c}=\theta_{N} \cong 8.5 \mathrm{k}$ agrees well with the experimental results. The value of superconducting gap $\frac{2 \Delta_{0}}{K_{B} T_{c}} \cong 3.54$ and $\frac{2 \Delta_{0}}{k_{B} T_{N}} \cong 4.5$ is comparable to that of BCS value. 
Fig-3 shows the plot of superconducting and antiferromagnetic order parameter for $\mathrm{DyNi}_{2} \mathrm{~B}_{2} \mathrm{C}$. The graph shows $T_{N}$ greater than $T_{c}$ which matches with experimental results. The value of critical temperature parameter $\theta_{c}$ and $\theta_{N}$ lies in the range 0.0022 to 0.0038 (corresponding to $T_{c} \sim 6.35$ to 10 ) and 0.0044 ( corresponding to $T_{N} \sim 12.78$ ) respectively.Similarly, the value of superconducting gap parameter obtained is in the range of 5.48 which is higher than BCS value. On the other hand, antiferromagnetic order parameter obtained is very small. In the coexisting region, the large fluctuation is observed.

Fig-4 represents the plot of superconducting and antiferromagnetic order parameter for $\mathrm{ErNi}_{2} \mathrm{~B}_{2} \mathrm{C}$ with temperature by varying external magnetic field than by varying hybridization potential.The critical temperature parameter $\theta_{c}$ and Neel temperature parameter $\theta_{N}$ remain in the range 0.0022-0.0026(corresponding to $T_{c} \sim 6.5$ to 7.5 ) and $T_{N}$ in the range of $0.0020-0.22$ ( corresponding to $T_{N} \sim$ 5.7 to 7).The value of $\frac{2 \Delta_{0}}{K_{B} T_{c}} \cong 4.2$ and $\frac{2 \Delta_{0}}{k_{B} T_{N}} \cong .0 .35$. The $T_{c}$ value decreases with increasing magnetic field and also with $\mathrm{v}$. The antiferromagnetic gap reducesby a large amount by increasing hybridization and also with external magnetic field.

Fig-5 shows the plot of order parameters for $\mathrm{TmNi}_{2} \mathrm{~B}_{2} \mathrm{C}$.The graphs show similar trends and for this compound $T_{C}>T_{N}$. The critical temperature and Neel temperature remains in the range of 7.5 and 7.0 respectively.The value of $\frac{2 \Delta_{0}}{K_{B} T_{c}} \cong 3.427$ and $\frac{2 \Delta_{0}}{k_{B} T_{N}} \cong 0.65$.The $T_{c}$ value decreases with increasing magnetic field and also with $\mathrm{v}$. The antiferromagnetic gap reduces by a large amount by increasing hybridization and also with external magnetic field.

The results shown above suggests the coexistence of superconducting and antiferromagnetic states.It is observed that when hybridization increases both superconducting and antiferromagnetic gap reduces for all the compounds. Hence we conclude that hybridization plays an important role in changing the superconducting transition temperature and gap parameter. The charge carriers appear to a couple very strongly to the spin system.Similarly,by increasing magnetic field, both transition and Neel temperature reduces throughout the range.
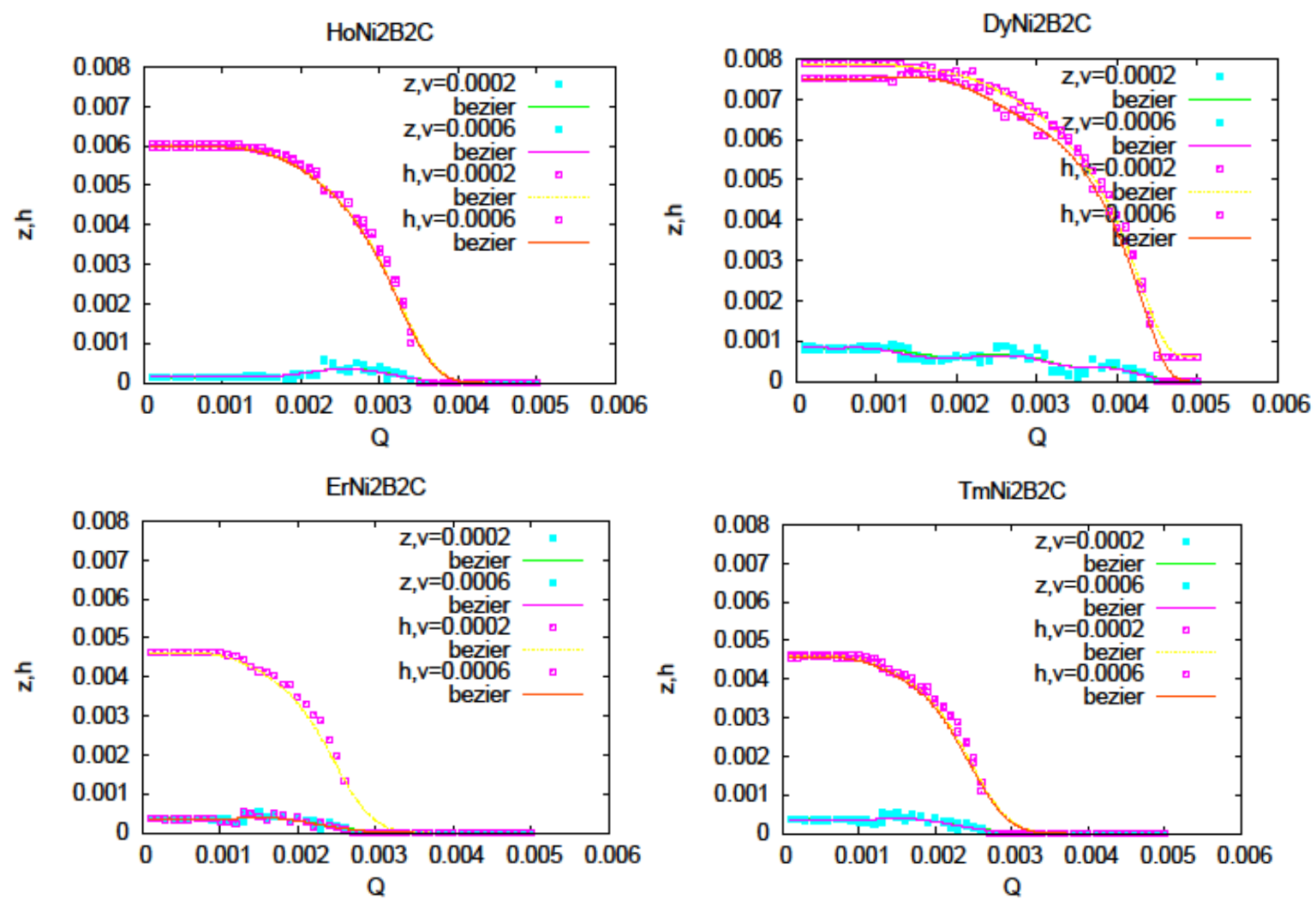
Figure 1.

In Fig-1 the temperature dependence of SC gap (z) and AFM gap (h) in the co-existing phase in absence of magnetic field for a small value of hybridization $(\mathrm{v})$ is considered.
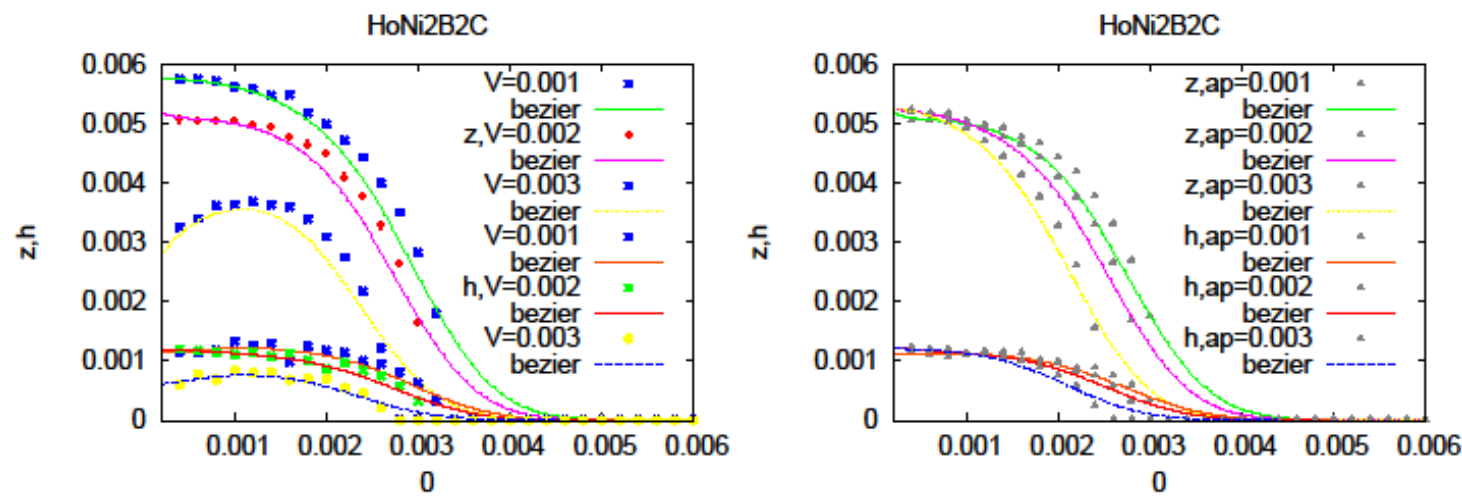

(a) Graph for different values of hybridization, $V \quad$ (b)Graph for different values of external magnetic field, $\mathrm{a}_{\mathrm{p}}$ Figure 2.

In Fig-2 the temperature dependence of SC gap (z) and AFM gap (h) in the co-existing phase in presence of magnetic field for $\mathrm{HoNi}_{2} \mathrm{~B}_{2} \mathrm{C}$ is considered.
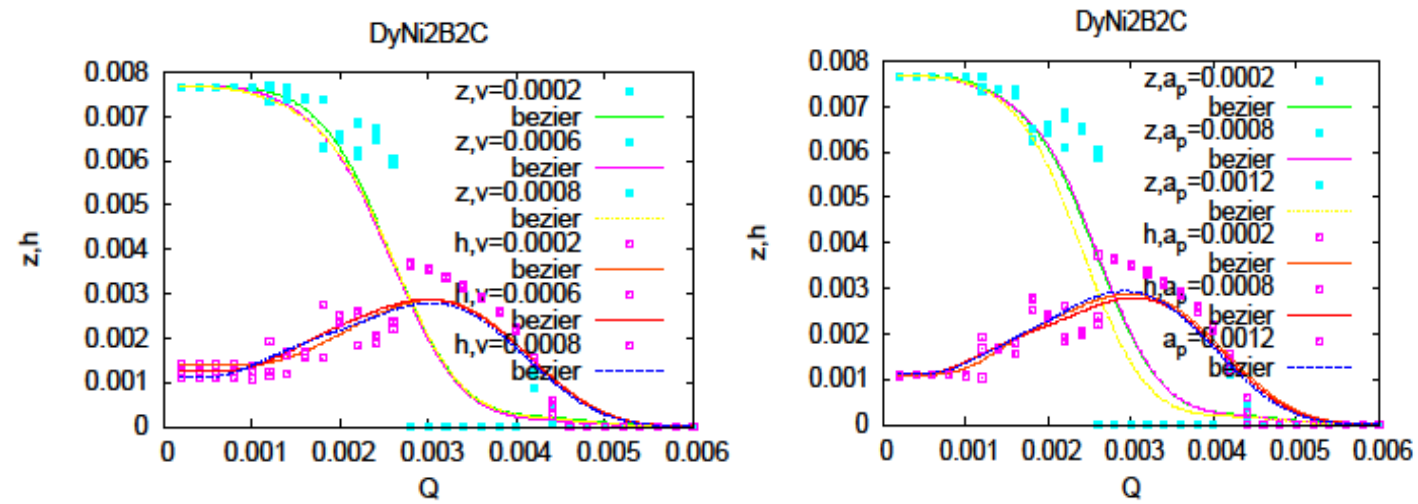

(a) Graph for different values of hybridization, $\mathrm{V}$ field, $a_{p}$

Figure 3

In Fig-3 the temperature dependence of SC gap (z) and AFM gap (h) in the co-existing phase in presence of magnetic field for $\mathrm{DyNi}_{2} \mathrm{~B}_{2} \mathrm{C}$ is considered.

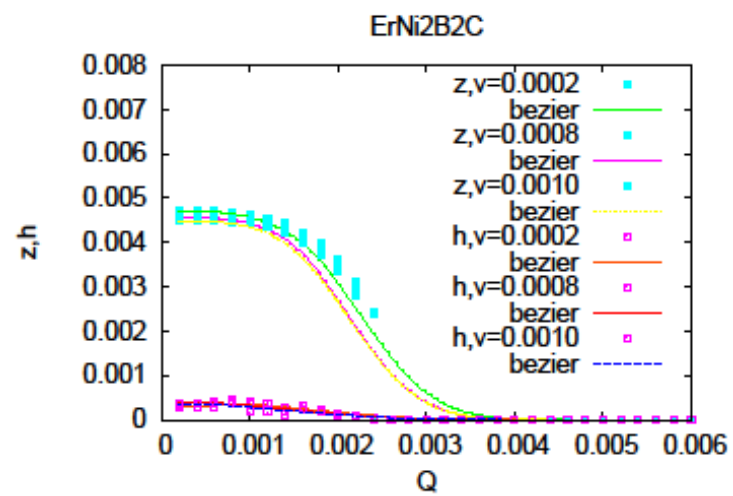

(a) Graph for different values of hybridization, $\mathrm{V}$ field, $\mathrm{a}_{\mathrm{p}}$

In Fig-4 the temperature dependence of SC gap (z) and AFM gap (h) in the co-existing phase in presence of magnetic field for $\mathrm{ErNi}_{2} \mathrm{~B}_{2} \mathrm{C}$ is considered.
ErNi2B2C

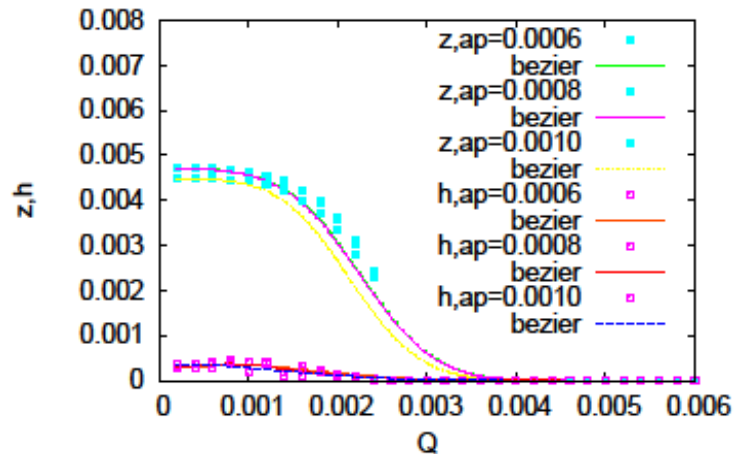

(b) Graph for different values of external magnetic
Figure 4. 


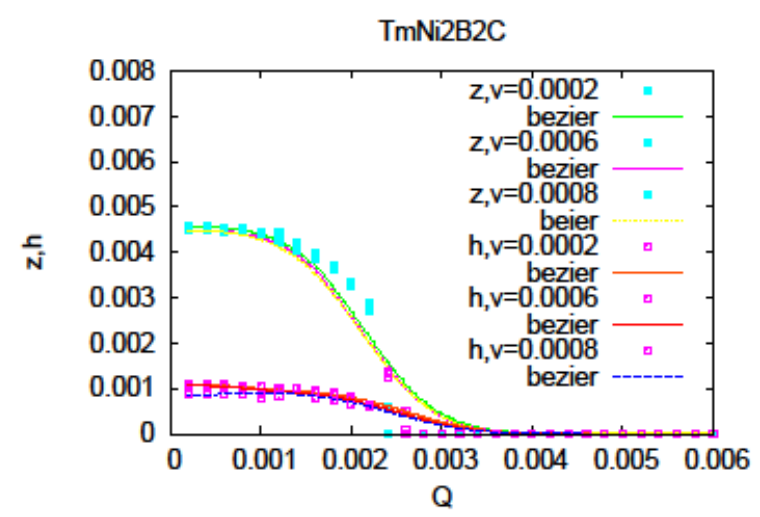

(a) Graph for different values of hybridization, $\mathrm{V}$

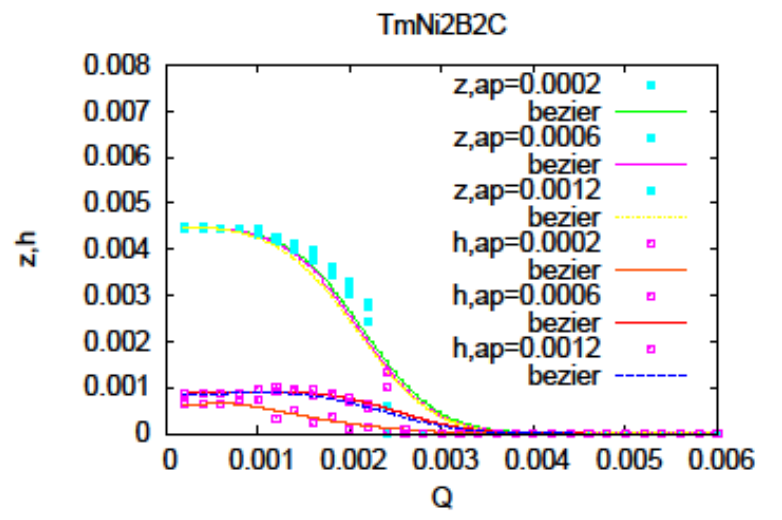

(b) Graph for different values of external magnetic field, $\mathrm{a}_{\mathrm{p}}$

Figure 5 .

In Fig-5 the temperature dependence of SC gap (z) and AFM gap (h) in the co-existing phase in presence of magnetic field for $\mathrm{T} \mathrm{mNi}_{2} \mathrm{~B}_{2} \mathrm{C}$ is considered.

\section{Conclusion}

In our model, we study the coexistence of superconducting and antiferromagnetic state for various rare earth compounds within the mean field approximation. We have taken a set of suitable, realistic model parameters. In our calculation, we have included the hybridization by taking different values of the external magnetic field. Our result shows the combination of $\theta_{c}$ and $\theta_{N}$ for different rare earth nickel borocarbide compounds.The values of $T_{c}$ and $T_{N}$ obtained for different compounds is in decreasing order with increasing magnetic field and hybridization. Due to large fluctuation, we could not get good results for $\mathrm{DyNi}_{2} \mathrm{~B}_{2} \mathrm{C}$ and $\mathrm{TmNi}_{2} \mathrm{~B}_{2} \mathrm{C}$ compound. Our calculated data agrees well with the experimental results.

\section{Acknowledgements}

We gratefully acknowledge Prof. S. Nayak for useful discussions and valuable suggestion to complete the work.

\section{References}

[1]. Fulde F, Zenui V, Zuricknagl G. Z.Phys B92 133; 1993. Fulde F. J Low Temp Phys $9545 ; 1994$.

[2]. Mazumdar C, Nagarajan R, Godart G, Gupta LC, Latroche M, Dhar SK, Levy Clement C, Padalia DD, Vijayaraghavan R. Solid State Communications 87 413; 1993.

[3]. Nagarajan R. et al Phy Rev. Lett 72 174; 1994.

[4]. Carfield PC, Gammel PL, Biship DJ. Physics Today 51 40; October 1998.

[5]. Behera SN, Panda BN, Rout GC, Entel P. International Journal of Modern Physics B15 2519; 2001.

[6]. Cho BK, Carfield PC, Hohnston DC. Phys Rev Lett 77, 163; 1996.

[7]. Ramakrishnan TV, Varma CM. Phy Rev B24 137; 1981.

[8]. Picket WF, Singh DJ. Phy Rev Lett 72 3702; 1994.

[9]. Matthews LF. Phy Rev B49 13279; 1994.

[10]. Stassis C, Bullock M, Zarestky J, Carfield PC, Goldman AI, Shirane G, Shapier SM. Phy Rev B55 R 8678 ; 1997.

[11]. Rhee JY, Wang M, Harman BN. Phy Rev B51 15585; 1995 - I.

[12]. Goldman, Stassis C, Carfield PC, Zarestky J, Dervenagas P, Cho BK. Phy Rev B50 9668; 1994.

[13]. Rout GC, Panda BN, Behera SN. Physics C333 104; 2000.

[14]. Zubarer DN. Son Phys Usp 95 7; 1960.

[15]. Behera SN, Panda BN, Rout GC, Entel P. Int. J. Mod. Phys. B 15, 2519; 2001. Sahu BK, Panda BN, Mod. Phys. Lett. B 24, 2879; 2010.

[16]. Das Salila, Padhi PC. Internal Journal of Modern Physics B, Vol. 30, No.8, 1650044; 2016.

[17]. Anderson PW, suhl GTH. Magnetism,vol.1.Academic Press, New York; 1963.

[18]. Hubbard. J.Proc.R. Soc. Lond. Ser. A. Math. Phys. Sci. 276.238; 1963.

[19]. Amici A, Thalmeier P. Journal of Low-Temperature Physics, Vol. 117. Nos. 516; 1999.

[20]. Naidyuk YUG, Behr G, Bobrov NL, Chernobay VN, Drechsler SL, Fuchs G, Kvitnitskaya OE, Naugle DG, Rathnayaka KDD, Yanson IK. Journal of Physics:Conference Series, 150, 052178; 2009. 
[21]. Rybatchenka LF. Physica B, 218, 189; 1996.

[22]. Yansona IK, Bobrova NL, Tomy CV, Paul DMcK Physica C 334 152; 2000.

[23]. Rybaltchenko LF. et al Europhysics Lett, 33, 483; 1996.

[24]. Sahu BK, Panda BN, Pramana 77, 4, 715;2015 Sahu BN, Panda BN ,Physica C 333, 104; 2015.

\section{Appendix}

The Greens function of Zubare [14] type is used in the calculation. Here we have used $A_{i}(\mathbf{k}, \omega), B_{i}(\mathbf{k}, \omega), C_{i}(\mathbf{k}, \omega)$ and $D_{i}(\mathbf{k}, \omega)(i=1,2)$, which are given as

$A_{1}(\mathrm{k}, \omega)=\left\langle\left\langle a_{k \uparrow} ; a_{k \uparrow}^{\dagger} \gg_{\omega}\right.\right.$

$A_{2}(\mathrm{k}, \omega)=\left\langle\left\langle a_{-k \downarrow}^{\dagger} ; a_{k \uparrow}^{\dagger}\right\rangle_{\omega}\right.$

$A_{3}(\mathrm{k}, \omega)=\left\langle\left\langle b_{k \uparrow} ; a_{k \uparrow}^{\dagger}\right\rangle_{\omega}\right.$

$A_{4}(\mathrm{k}, \omega)=\left\langle b_{-k \downarrow}^{\dagger} ; a_{k \uparrow}^{\dagger} \gg_{\omega}\right.$

$A_{5}(\mathrm{k}, \omega)=\left\langle\left\langle f_{1, k \uparrow} ; a_{k \uparrow}^{\dagger}\right\rangle_{\omega}\right.$

$A_{6}(\mathrm{k}, \omega)=\left\langle\left\langle f_{1,-k \downarrow}^{\dagger} ; a_{k \uparrow}^{\dagger}\right\rangle_{\omega}\right.$

$A_{7}(\mathrm{k}, \omega)=\left\langle\left\langle f_{2, k \uparrow} ; a_{k \uparrow}^{\dagger}\right\rangle_{\omega}\right.$

$A_{8}(\mathrm{k}, \omega)=\left\langle\left\langle f_{2,-k \downarrow}^{\dagger} ; a_{k \uparrow}^{\dagger} \gg{ }_{\omega}(19)\right.\right.$

$B_{1}(\mathrm{k}, \omega)=\left\langle\left\langle b_{k \uparrow} ; b_{k \uparrow}^{\dagger}\right\rangle_{\omega}\right.$

$B_{2}(\mathrm{k}, \omega)=\left\langle b_{-k \downarrow}^{\dagger} ; b_{k \uparrow}^{\dagger}\right\rangle_{\omega}$

$C_{1}(\mathrm{k}, \omega)=\left\langle\left\langle a_{k \downarrow} ; a_{k \downarrow}^{\dagger} \gg_{\omega}\right.\right.$

$D_{1}(\mathrm{k}, \omega)=\left\langle b_{k \downarrow} ; a_{k \downarrow}^{\dagger} \gg_{\omega}\right.$

The equation of motion and commutation relations of the fermion operators $a_{k, \sigma}, b_{k, \sigma}, f_{k, \sigma}$ are used to evaluate the above Green's function $A_{i}(k, \omega)$ (i=1-6) which are

$$
\begin{aligned}
& {\left[\omega-\left(\varepsilon_{F}+\frac{1}{2} g \mu_{B} B\right)-\frac{h}{2}\right] A_{1}(\omega)=\frac{1}{2 \pi}+\varepsilon_{0}(\mathbf{k}) A_{3}(\omega)+V A_{5}(\omega)-\Delta A_{2}(\omega)} \\
& {\left[\omega+\left(\varepsilon_{F}-\frac{1}{2} g \mu_{B} B\right)-\frac{h}{2}\right] A_{2}(\omega)=-\varepsilon_{0}(\mathbf{k}) A_{4}(\omega)-V A_{6}(\omega)-\Delta A_{1}(\omega)} \\
& {\left[\omega-\left(\varepsilon_{F}-\frac{1}{2} g \mu_{B} B\right)+\frac{h}{2}\right] A_{3}(\omega)=\varepsilon_{0}(\mathbf{k}) A_{1}(\omega)+V A_{7}(\omega)-\Delta A_{4}(\omega)} \\
& {\left[\omega+\left(\varepsilon_{F}+\frac{1}{2} g \mu_{B} B\right)+\frac{h}{2}\right] A_{4}(\omega)=-\varepsilon_{0}(\mathbf{k}) A_{2}(\omega)-V A_{8}(\omega)-\Delta A_{3}(\omega)} \\
& \left.\left.\left[\begin{array}{l}
\omega \\
{[}
\end{array}\right] \varepsilon_{f}+\frac{1}{2} g \mu_{B} B\right)\right] A_{7}(\omega)=V A_{3}(\omega) \\
& {\left[\omega+\left(\varepsilon_{f}-\frac{1}{2} g \mu_{B} B\right)\right] A_{8}(\omega)=-V A_{4}(\omega)} \\
& {\left[\omega-\left(\varepsilon_{f}+\frac{1}{2} g \mu_{B} B\right)\right] A_{5}(\omega)=V A_{1}(\omega)}
\end{aligned}
$$




$$
\left[\omega+\left(\varepsilon_{f}-\frac{1}{2} g \mu_{B} B\right)\right] A_{6}(\omega)=-V A_{2}(\omega)
$$

Similarly, we can write for B, C, D The Fermi level is taken in the middle of the antiferromagnetic band gap and the Fermi level is taken as zero. Here the localized f energy level coincides with the Fermi level. We have considered the half-filled band.The symbols taken in the equation are After solving the coupled equations obtained from above equations we find out the green's functions $A_{1.2}(k, \omega), B_{1,2}(k, \omega), C_{1}(k, \omega)$ and $D_{1}(k, \omega)$ as

$$
\begin{aligned}
& A_{1}=\frac{1}{4 \pi}\left[\frac{M-\omega^{\prime} \Delta_{-}}{D_{1}}+\frac{M+\omega^{\prime} \Delta_{+}}{D_{2}}\right] \\
& A_{2}=\frac{1}{4 \pi}\left[\frac{M-\omega^{\prime} \Delta_{-}}{D_{1}}-\frac{M+\omega^{\prime} \Delta_{+}}{D_{2}}\right] \\
& B_{1}=\frac{1}{4 \pi}\left[\frac{M-\omega^{\prime} \Delta_{+}}{D_{2}}+\frac{M+\omega^{\prime} \Delta_{-}}{D_{1}}\right] \\
& B_{2}=\frac{1}{4 \pi}\left[\frac{M-\omega^{\prime} \Delta_{+}}{D_{2}}-\frac{M+\omega^{\prime} \Delta_{-}}{D_{1}}\right] \\
& C_{1}=\frac{1}{4 \pi}\left[\frac{M_{1}+\omega^{\prime \prime} \Delta_{-}}{D_{1}^{\prime}}+\frac{M_{1}+\omega^{\prime \prime} \Delta_{+}}{D_{2}^{\prime}}\right] \\
& D_{+}=\Delta+\frac{h}{2} ; \Delta_{-}=\Delta-\frac{h}{2} \\
& D_{1,2}=\omega^{\prime 4}-\omega^{\prime 2}\left[\varepsilon_{0}^{2}(k)+\Delta_{\mp}^{2}+2 V^{2}\right]+V^{4} \\
& D_{1}=\frac{1}{4 \pi}\left[\frac{M_{1}+\omega^{\prime \prime} \Delta_{+}}{D_{2}^{\prime}}+\frac{M_{1}+\omega^{\prime \prime} \Delta_{-}}{D_{1}^{\prime}}\right] \\
& M_{1}^{\prime 2}(k)+\omega^{\prime \prime 2}\left[\varepsilon_{0}^{2}(k)+\Delta_{ \pm}^{2}+2 V^{2}\right]+V^{4}{ }^{2}(k)+V^{4} \\
& D_{1,2}^{2}
\end{aligned}
$$


where

$$
\omega^{\prime}=\omega-\frac{1}{2} g \mu_{B} B=\omega-\frac{\alpha}{2}
$$

and $\omega^{\prime \prime}=\omega+\frac{1}{2} g \mu_{B} B=\omega+\frac{\alpha}{2}$

$$
\alpha=g \mu_{B} B
$$

The quasi particle energy bands can be obtained from the poles of the Green's function i,e $\omega_{i \pm}(1$ to 4$)$

$$
D_{1,2}=0 \text { and } D_{1,2}^{\prime}=0
$$

